\title{
Influencia del alga Ascophyllum nodosum como promotor de crecimiento en la etapa de alevinaje de la trucha arco iris (Oncorhynchus mykiss)
}

\section{(Influence of the seaweed Ascophyllum nodosum as a growth promoter in the stage of rainbow trout (Oncorhynchus mykiss))}

\author{
Diego López ${ }^{1}$, Iván Naranjo², Olga Pérez², Vinicio Uday³
}

\begin{abstract}
Resumen:
Se evaluó la calidad del alga Ascophyllum nodosum, como promotor de crecimiento en la etapa de alevinaje de la Trucha Arco Iris (Oncorhynchus mykiss) y su efecto inmunoestimulante respecto a enfermedades micóticas como la Saprolegnia sp. Se utilizaron 6000 alevines de 77 días de edad, con un peso promedio de $0,15 \mathrm{~g}$, distribuidos bajo un DCA. Para el análisis de resultados se aplicó el análisis de varianza y separación de medias (Duncan al $p<0,05$ ), comparaciones ortogonales y chi cuadrado. El alga Ascophyllum nodosum adicionada como suplemento alimenticio en la etapa de crecimiento con un nivel del $2 \%$ y $98 \%$ de alimento concentrado fue el que mejores resultados zootécnicos y económicos alcanzó: peso inicial 0,84 g, ganancia diaria de peso 0,30 g, peso final 34,60 g. conversión alimenticia 0,19, mortalidad $4,60 \%$, y el costo de producción por kilo de carne es $\$ 35,24$. Respecto al desafío micótico (hongo Saprolegnia sp.), la administración del alga Ascophyllum nodosum en los porcentajes de $2 \%, 2,5 \%$ y $3 \%$ como suplemento alimenticio, proporcionó resistencia a los alevines.
\end{abstract}

Palabras clave: alga; suplemento; alevines; crecimiento; hongo.

\begin{abstract}
:
The quality of the algae Ascphyllum nodosum was assessed as a growth promoter in the breeding stage of Rainbow Trout (Oncorhynchus mykiss) and its immunostimulant effect with regard to fungal diseases as the Saprolegnia sp. 6000 fingerlings of 77 days of age were used, with an average weight of $0.15 \mathrm{~g}$, distributed under a DCA. For the analysis of the results, it was applied the analysis of variance and separation of averages (Duncan to $\mathrm{p}<0.05$ ), orthogonal comparisons and chi square. The seaweed Ascophyllum nodosum added as nutritional supplement in the growth stage with a level of $2 \%$ and $98 \%$ of concentrated food was the one that reached the best zoo technical and economic results: initial weight $0.84 \mathrm{~g}$, daily weight gain $0.30 \mathrm{~g}$, final weight $34.60 \mathrm{~g}$., nutritive conversion 0.19 , mortality $4.60 \%$, and the cost of production per kilo of meat is $\$ 35.24$. Regarding the mycotic challenge (fungus Saprolegnia sp.), the administration of the seaweed Ascophyllum nodosum on the percentages of $2 \%$, $2.5 \%$ and $3 \%$ as nutritional supplement, provided resistance to the fingerlings.
\end{abstract}

Keywords: fingerlings; seaweed; growth; fungus; supplement.

\footnotetext{
${ }^{1}$ Universidad Central del Ecuador, Quito - Ecuador

2 Universidad Tecnológica Equinoccial, Quito - Ecuador (\{nsij505711, operez\} @ute.edu.ec)

3 Universidad de las Fuerzas Armadas, Sangolquí - Ecuador (mvuday@espe.edu.ec)
} 


\section{Introducción}

Según la FAO (2017) existen cerca de 567 especies acuáticas que se cultivan actualmente en todo el mundo, lo que representa una enorme riqueza de diversidad genética dentro y entre las especies. La acuicultura la practican tanto los agricultores pobres de los países en desarrollo como las empresas multinacionales.

Comer pescado forma parte de la tradición cultural de muchas personas y, en términos de beneficios para la salud, tiene un excelente perfil nutricional. Es una buena fuente de proteínas, ácidos grasos, vitaminas, minerales y micronutrientes esenciales. El 80 por ciento de la producción acuícola actual deriva de animales que se encuentra en la parte inferior en la cadena alimentaria, como peces omnívoros y herbívoros y moluscos. $\mathrm{Si}$ se tiene en cuenta su comportamiento dinámico en los últimos 30 años y la disminución de la pesca de captura, es probable que el crecimiento futuro del sector pesquero derive principalmente de la acuicultura (FAO, 2017)

En cambio, las pesquerías del interior, y dentro de ellas en particular la producción intensiva de peces en estanques, pilas y similares, ofrecen a este respecto posibilidades ostensiblemente mayores. Desde mediados de la década de los sesenta se registra en el ámbito internacional un tipo de producción denominada Acuicultura, en la cual se engloba por lo común el cultivo masivo de los peces o producción industrial de los mismos en aguas del interior y también en aguas costeras. Sus orígenes hay que buscarlos en la cría de peces en estanques, que en numerosos países reviste gran importancia económica y tiene una larga tradición (CENTRO EGIPCIO INTERNACIONAL PARA LA AGRICULTURA, 1994).

En los estanques los peces se alimentan primariamente con organismos acuáticos inferiores, y con carácter secundario a base de una alimentación adicional más o menos abundante constituida por diversos productos vegetales o animales (Conroy, 1991).

El uso regular de la harina de Kelp de Ascophyllum nodosum como suplemento alimenticio, ayuda a mejorar el aprovechamiento de todos los ingredientes en una ración completa de alimento, mejorando la salud y rendimiento general de sus animales, es ampliamente utilizada en la acuacultura, como un agente adherente que ayuda en la reducción de desechos en alimentos, extruidos o granulados, para peces. Adicionalmente, a menudo se observa una mejora considerable en la salud de los peces. El Kelp es también ampliamente utilizado en alimentos para camarones y en las dietas de los mariscos (ACADIAN SEAPLANTS, 2017).

Según Summer Zone (2017) actualmente en el Ecuador este suplemento alimenticio es utilizado para la alimentación del camarón, ya que en esta especie se ha conseguido desarrollar una mayor ganancia de peso. En lo que se refiere a la agricultura este suplemento es utilizado como abono orgánico, consiguiendo buenos resultados respecto a otros abonos orgánicos de origen animal e incluso vegetal.

La saprolegniosis es provocada por ficomicetos pertenecientes al orden Saprolegniales, entre los cuales son de mayor importancia las especies de Saprolegnia y Achlya, la mayoría de estos hongos son saprofitos, pero existen determinadas especies que pueden considerarse como ictiopatógenas. Entre dichas especies figuran: Saprolegnia mixta, Saprolegnia ferax, Saprolegnia parasitica, Saprolegnia monoica (esta última es responsable de infecciones intestinales), y Achlya flagellata. Un carácter muy importante de los saprolegniales, por ser ficomicetos, es que las hifas son coenociticas, o sea que no poseen tabiques, las hifas por lo general son delgadas, ramificadas y con un espesor de unos 20 micrones. La forma de reproducción puede ser sexual o asexual (Conroy, 1991).

Conroy (1991), se refiere que en casos normales de saprolegniasis, las hifas están rodeadas por las células cutáneas, las cuales vienen a constituir el sustrato para el hongo. Una vez destruido el tegumento, el hongo penetra la musculatura y finalmente llega a los órganos viscerales del pez. El desarrollo de las hifas en la piel y musculatura da lugar a la formación de zonas necróticas, las cuales contribuyen a la muerte del pez, 
salvo que la enfermedad sea controlada a tiempo. La saprolegniasis también es de importancia en cuanto a las branquias, ya que dan lugar a una importante necrosis de los lámelos de las mismas.

En el cultivo de salmónidos, la saprolegniasis adquiere singular importancia con respecto a la incubación de los huevos. El hongo se desarrolla en primer lugar sobre los huevos no fertilizados o dañados, a partir de los cuales se extienden hacia los huevos vivos, según parece, en condiciones normales el hongo no se desarrolla sobre huevos vivos, a menos que estos tengan algo de materia orgánica adherida a la superficie. En un lapso muy corto, las hifas llegan a formar una trama filamentosa que da lugar a la muerte de los huevos afectados por asfixia (Conroy, 1991).

Con estos antecedentes, el propósito de la presente investigación fue la de promover el crecimiento de la trucha Arco Iris (Oncorhynchus mykiss), por medio de la solución de kelp (alga Ascophyllum nodosum) utilizada como suplemento alimenticio, y a su vez conseguir una posible mayor resistencia a la micosis causada por el hongo Saprolegnia sp (moho de agua), patógeno que incide de manera común en la etapa de alevinaje de la trucha Arco iris en los núcleos de explotación intensiva, inclusive en el Centro de Investigaciones Acuícolas Papallacta (CENIAC-P).

Los objetivos que se trazaron en la investigación fueron: determinar la eficiencia de la solución de kelp (alga Ascophyllum nodosum) como suplemento alimenticio en la etapa de alevinaje de la trucha Arco Iris, establecer la mortalidad de los alevines en los diferentes tratamientos utilizados con la solución de kelp (alga Ascophyllum nodosum), determinar la posible mortalidad de los alevines, alimentados con la solución de kelp (alga Ascophyllum nodosum), al ser desafiados con el hongo Saprolegnia sp.

La investigación es muy importante para el Ecuador, pues al ser un estudio pionero no se ha encontrado literatura para discutir la información generada en este estudio, sin embargo, los resultados obtenidos permiten demostrar que existe la posibilidad de mejorar la producción de este tipo de pez en el Ecuador.

\section{Metodología}

\subsection{Localización de la investigación}

La investigación se llevó a cabo en el Centro de Investigaciones Acuícolas de Papallacta (CENIAC-P) ubicada en la provincia de Napo, cantón Quijos, parroquia Papallacta, barrio Llanura de Baños, ubicado a 3296 m.s.n.m., $0^{\circ} 29^{\prime}$ latitud sur y $78^{\circ} 10^{\prime}$ de longitud oeste. Temperatura ambiental máxima de $14^{\circ} \mathrm{C}$ y mínima de $6^{\circ} \mathrm{C}$. La calidad del agua que se utilizó presentó los siguientes parámetros:

Análisis Físico y Químico del agua

Temperatura: Máxima: $11^{\circ} \mathrm{C}$, Media: $9^{\circ} \mathrm{C}$, Mínima: $7^{\circ} \mathrm{C}$. Ph: 7,6; Sodio: 12; Cromo: 0,5 ppm; Aluminio: 0,1 ppm; Hierro: 0,1 ppm

\subsection{Evaluación productiva del alga Ascophyllum nodosum como promotor de crecimiento}

En la primera fase se estudió el rendimiento de la adición del alga Ascophyllum nodosum en el alimento balanceado, como promotor de crecimiento en la etapa de alevinaje.

Se emplearon alevines de trucha Arco Iris de 77 días desde su eclosión, los cuales fueron transportados desde la sala de incubación hasta el laboratorio de Biotecnología en donde fueron depositados en estanques de plástico: ancho 0,445 m, largo 0,640 m, altura $0,380 \mathrm{~m}$, altura de agua $0,27 \mathrm{~m}$. Para cada tratamiento (cuatro) y repeticiones (3) se utilizaron 500 peces, dando un total de 6000 animales.

La cantidad de alimento suministrado a los peces se la calculó a base de la tabla de Leitriz y la frecuencia de alimentación fue de seis veces al día, fraccionada en tres 
raciones por la mañana y tres por la tarde. La determinación del peso de los animales se la efectuó cada quince días. La medición de oxígeno disuelto, $\mathrm{pH}$ y temperatura, así como la limpieza de los estanques y medición de la mortalidad, se la efectuó diariamente.

La bioseguridad fue un factor importante para la investigación, se colocó un pediluvio a la entrada del laboratorio para la desinfección del calzado de las personas que ingresen al laboratorio, así como la ubicación de un rociador para la desinfección de manos (Imaki, 1987).

En la Tabla 1 se presentan los tratamientos utilizados en la investigación.

Tabla 1. Descripción de los tratamientos utilizados en el estudio de la influencia del alga Ascophyllum nodosum como promotor de crecimiento en la etapa de alevinaje de la trucha Arco Iris (Oncorhynchus mykiss).

\begin{tabular}{lll}
\hline Tratamiento & Código & Descripción \\
\hline Testigo & T & Solo alimento \\
Tratamiento 1 & E1 & $2 \%$ de alga con un $98 \%$ de alimento \\
Tratamiento 2 & E2 & $2,5 \%$ de alga con un $97,5 \%$ de alimento \\
Tratamiento 3 & E3 & $3 \%$ de alga con un $97 \%$ de alimento \\
\hline
\end{tabular}

\subsection{Evaluación del efecto inmunoestimulante del alga Ascophyllum nodosum ante desafíos micóticos (Saprolegnia sp).}

En la segunda fase se estudió la respuesta inmunitaria de los alevines de trucha Arco Iris alimentados con el alga Ascophyllum nodosum y alimento concentrado, al ser inoculados por vía oral con la cepa pura del hongo Saprolegnia sp. Para el desafío con el hongo Saprolegnia sp., se emplearon muestras de peces, ovas y del agua que contenían dicho patógeno.

Asilamiento.- La obtención de la cepa pura del hongo Saprolegnia sp. se la realizó en el laboratorio de Microbiologia del CENIAC-P por medio del aislamiento y del crecimiento de las muestras obtenidas de ovas contaminadas con dicho hongo, las mismas que fueron colocadas en cajas Petri, que contenían Agar Sabouraud (medio universal para el crecimiento de hongos). Para un crecimiento óptimo del hongo Saprolegnia sp., se recomendó utilizar una temperatura de $20^{\circ} \mathrm{C}$ por un período de siete días en la estufa, con el fin de conseguir un desarrollo homogéneo del hongo en la superficie del medio de cultivo (Nilubol y Yuasa, 1998). La Figura 1 muestra la afección de Saprolegnia sp., a las ovas y su morfología.
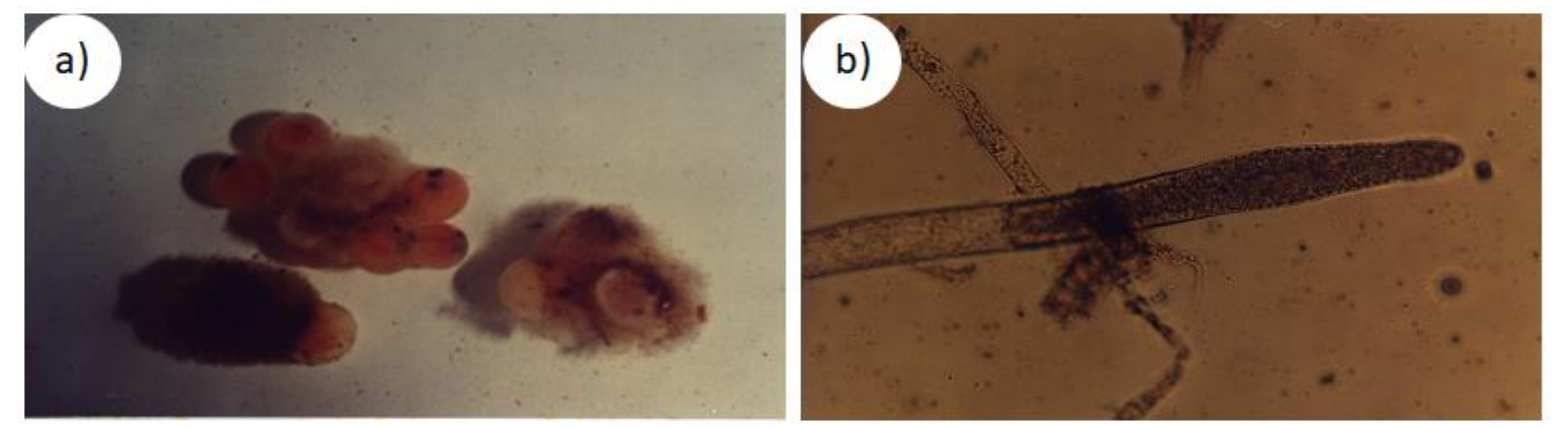

Figura 1. a). Ovas afectadas por Saprolegnia sp. (Imaki, 1987). b). Morfología del hongo Saprolegnia sp. (Niwa y Zeballo, 1990).

Tipificación.- Por medio de la utilización de agujas de disección, se procedió a tomar unas cuantas colonias del hongo Saprolegnia sp., del medio del cultivo, las cuales son colocadas en un portaobjetos. Para una mejor tipificación del hongo, las colonias son extendidas en toda la superficie del portaobjeto y a la vez se adiciona una gota de agua destilada, consiguiéndose de esta manera una observación correcta de la morfología del 
hongo, con lo cual se evitaron posibles confusiones en la determinación de este patógeno respecto a otros (Nilubol y Yuasa, 1998).

Preparación de la solución de PBS.- La preparación de la solución de PBS se la obtuvo al colocar $0,96 \mathrm{~g}$ de PBS (polvo) en 100 mililitros de agua destilada, mezcla que a su vez se trasvasó en tres vasos Erlenmeyer, los cuales fueron herméticamente sellados con papel aluminio y a su vez colocados en la estufa, con la finalidad de obtener una mayor pureza de la solución de PBS por medio de la esterilización para eliminar cualquier agente contaminante (Niwa y Zeballo, 1990).

Preparación del inóculo.- La preparación del inóculo consistió en pesar $0 \mathrm{~g}, 0,05 \mathrm{~g}$, $0,01 \mathrm{~g}$ de colonias de Saprolegnia sp., aislada y tipificada, las mismas que fueron añadidas a la solución de PBS esterilizada $(100 \mathrm{ml})$, mezcla que a su vez se homogenizó por un minuto, hasta observar una coloración blanquecina (Niwa y Zevallo, 1990) (Figura 2).
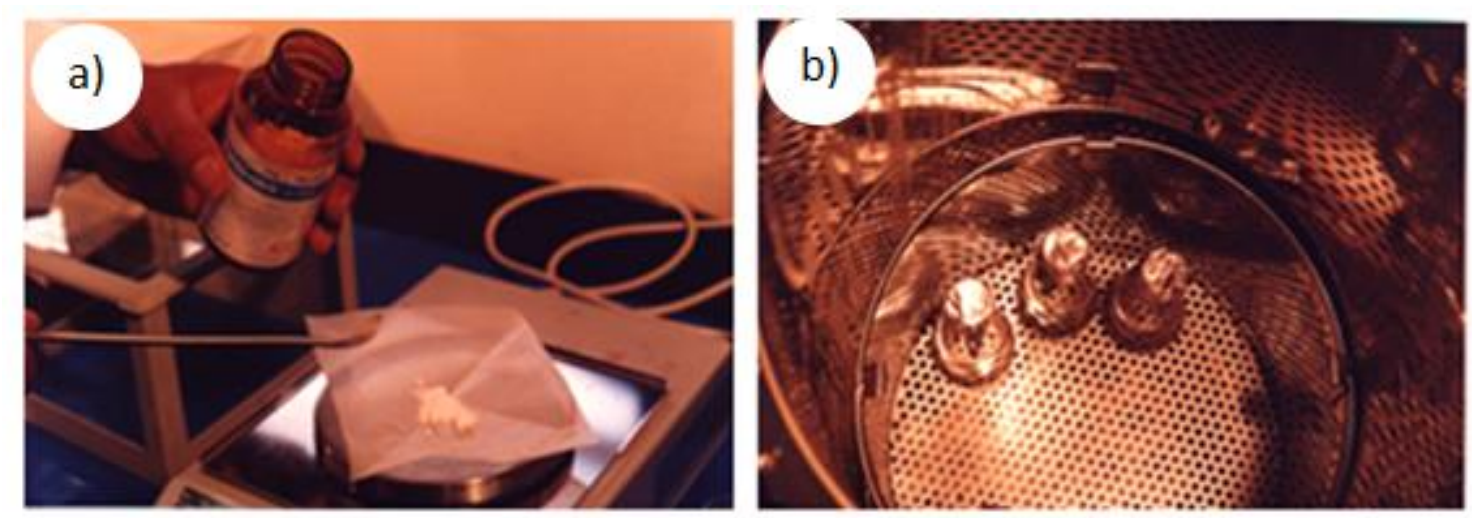

Figura 2. a) Preparación de la solución PBS b) Esterilización de la solución PBS, Niwa y Zeballo, 1990).

Inoculación.- En cuanto a la inoculación del agente patógeno, se la realizó por vía oral en una cantidad de $0,1 \mathrm{ml}$ por medio de una sonda que ingresó por la cavidad oral hasta el intestino del pez. Previamente a la inoculación de la enfermedad, los peces consiguieron un peso de mayor de un gramo y una edad de 187 días. Para la inoculación se tomó el $10 \%$ de la población de cada tratamiento. Posteriormente a la inoculación se midió a partir de las 24 horas y por 15 días, el índice de mortalidad que pudo existir por cada tratamiento (Niwa y Zevallo, 1990).

\subsection{Diseño experimental}

Las unidades experimentales durante la etapa de evaluación productiva del alga como promotor de crecimiento, se distribuyeron bajo un diseño completamente al azar, con tres repeticiones. La separación de medias fue con Duncan al nivel de $P \leq 0,05$. En las variables peso inicial, ganancia diaria, peso final y conversión alimenticia se realizaron comparaciones ortogonales; la mortalidad se aplicó la prueba chi cuadrado con $\mathrm{P} \leq 0,05$. Los resultados experimentales obtenidos fueron analizados con el software estadístico Infostat versión 2016 (Di Rienzo, et al. 2016).

\subsection{Mediciones experimentales}

\subsubsection{Evaluación productiva}

Se determinaron variables como: peso inicial, ganancia diaria, peso final, conversión alimenticia, mortalidad y análisis del costo de producción por kg de carne producida. 


\subsubsection{Evaluación inmunoestimulante del alga Ascophyllum nodosum}

Se midió el porcentaje de mortalidad diaria de peces inoculados con el hongo Saprolegnia sp.

\section{Resultados y Discusión}

\subsection{Evaluación productiva del alga Ascophyllum nodosum como promotor de crecimiento en la etapa de alevinaje de la trucha Arco Iris}

Los resultados del análisis de varianza y de prueba de separación de medias obtenidos en el estudio de la influencia del alga Ascophyllum nodosum como suplemento alimenticio en alevinaje de la trucha Arco Iris se reportan en las Tabla 2 y Tabla 3 respectivamente.

Tabla 2. Análisis de varianza para las variables peso inicial (g), ganancia diaria (g), peso final (g) y conversión alimenticia, dentro del estudio influencia del alga Ascophyllum nodosum como promotor de crecimiento en la etapa de alevinaje de la trucha Arco Iris Oncorhynchus mykiss.

\begin{tabular}{|c|c|c|c|c|c|}
\hline $\begin{array}{l}\text { Fuentes de } \\
\text { variación }\end{array}$ & $\begin{array}{c}\text { Grados de } \\
\text { libertad }\end{array}$ & $\begin{array}{c}\text { Peso inicial } \\
\text { (g) }\end{array}$ & $\begin{array}{c}\text { Ganancia diaria } \\
\text { (g) }\end{array}$ & $\begin{array}{c}\text { Peso final } \\
\text { (g) }\end{array}$ & $\begin{array}{l}\text { Conversión } \\
\text { alimenticia }\end{array}$ \\
\hline Tratamiento & & 0,16 * & $0,01^{*}$ & \multicolumn{2}{|c|}{$115,95 * 0,000624$ * } \\
\hline T vs E1, E2, E3 & 1 & $0,12 \mathrm{~ns}$ & $0,00011 \mathrm{~ns}$ & \multicolumn{2}{|c|}{0,67 ns 0,001667 * } \\
\hline $\mathrm{E} 1$ vs $\mathrm{E} 2, \mathrm{E} 3$ & 1 & 0,29 * & 0,02 * & \multicolumn{2}{|c|}{$320,05^{*} 0,000001 \mathrm{~ns}$} \\
\hline E2 VS E3 & 1 & $0,09 \mathrm{~ns}$ & 0,0024 ns & \multicolumn{2}{|c|}{27,14 * } \\
\hline Error & 44 & 0,03 & 0,00017 & \multicolumn{2}{|c|}{2,21} \\
\hline Total & 47 & & & & \\
\hline \multicolumn{2}{|c|}{ Coeficiente de variación \% } & 23,33 & 4,97 & 4,91 & 5,33 \\
\hline
\end{tabular}

Tabla 3. Efecto de la influencia del alga Ascophyllum nodosum como promotor de crecimiento en la etapa de alevinaje de la trucha Arco Iris Oncorhynchus mykiss en las variables peso inicial (g), ganancia diaria de peso (g), peso final (g), conversión alimenticia.

\begin{tabular}{lcccc}
\hline Tratamientos & $\begin{array}{c}\text { Peso inicial } \\
(\mathrm{g})\end{array}$ & $\begin{array}{c}\text { Ganancia diaria } \\
(\mathrm{g})\end{array}$ & $\begin{array}{c}\text { Peso final } \\
(\mathrm{g})\end{array}$ & $\begin{array}{c}\text { Conversión } \\
\text { alimenticia }\end{array}$ \\
\hline $\mathrm{T}$ & $0,83 \mathrm{~A}$ & $0,26 \mathrm{~B}$ & $30,11 \mathrm{~B}$ & $0,21 \mathrm{~A}$ \\
$\mathrm{E} 1$ & $0,84 \mathrm{~A}$ & $0,30 \mathrm{~A}$ & $34,60 \mathrm{~A}$ & $0,19 \mathrm{~B}$ \\
$\mathrm{E} 2$ & $0,59 \mathrm{~B}$ & $0,26 \mathrm{~B}$ & $29,33 \mathrm{~B}$ & $0,19 \mathrm{~B}$ \\
$\mathrm{E} 3$ & $0,71 \mathrm{AB}$ & $0,24 \mathrm{C}$ & $27,21 \mathrm{C}$ & $0,19 \mathrm{~B}$ \\
\hline
\end{tabular}

En la Figura 3 se presentan las comparaciones ortogonales significativas en el estudio de influencia del alga Ascophyllum nodosum como promotor de crecimiento en la etapa de alevinaje de la trucha Arco Iris Oncorhynchus mykiss.

\subsubsection{Peso inicial}

En la Tabla 1, se presentan valores obtenidos para los diferentes tratamientos y el testigo. Existen diferencias estadísticas entre los tratamientos $(p \leq 0,05)$, diferencia que fue corregida al momento de realizar el análisis de ganancia diaria de peso.

La comparación de medias con el método de Duncan indica que el tratamiento que empieza con mayor peso inicial, fue el $\mathrm{E} 1$ con un peso de $(0,84 \mathrm{~g})$ en relación con los demás tratamientos: T $(0,83 \mathrm{~g})$, E3 $(0,71 \mathrm{~g})$ y E2 $(0,59 \mathrm{~g})$. (Tabla 2). La comparación ortogonal E1 vs E2, E3 resultó significativa y corrobora al resultado obtenido con el método de Duncan. 


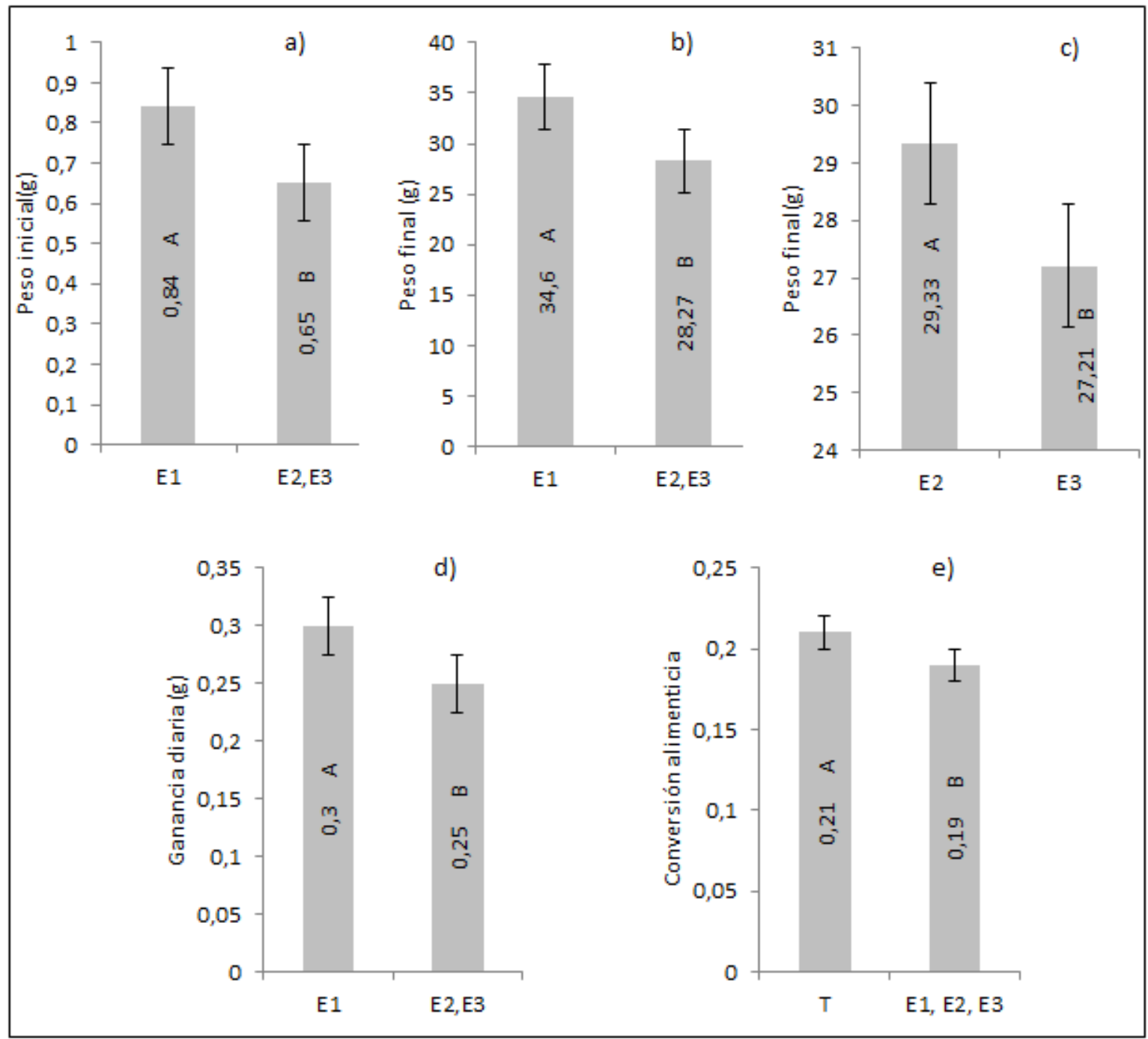

Figura 3. Comparaciones ortogonales significativas en el estudio de influencia del alga Ascophyllum nodosum como promotor de crecimiento en la etapa de alevinaje de la trucha Arco Iris Oncorhynchus mykiss. a) comparación ortogonal del peso inicial en gramos de E1 versus E2, E3; b) comparación ortogonal del peso final en gramos de E1 versus E2, E3; c) comparación ortogonal del peso final en gramos de E2 versus E4; d) comparación ortogonal de la ganancia diaria de peso en gramos de E1 versus E2, E3 y e) comparación ortogonal de la conversión alimenticia de $T$ versus el resto de tratamientos.

\subsubsection{Ganancia diaria de peso}

Estadísticamente se observan diferencias entre tratamientos en la variable ganancia de peso diaria $(p \leq 0,05)$. Así mismo la comparación ortogonal E1 vs E2, E3 presentó diferencias (Figura 3) donde sobresale en promedio de E1 con un valor de 0,30 $\mathrm{g}$ respecto al promedio conjunto de E2, E3. De acuerdo con Duncan E1 obtuvo la mayor ganancia diaria de peso $(0,30 \mathrm{~g})$; seguido por el tratamiento $\mathrm{T}(0,26 \mathrm{~g})$, a continuación el E2 $(0,26 \mathrm{~g})$ y por último el E3 $(0,24 \mathrm{~g})$ (Tabla 2$)$. Probablemente el tratamiento E1 ( $2 \%$ de alga) sea la cantidad adecuada, mejore la digestibilidad y logre incrementar el valor nutritivo del balanceado.

\subsubsection{Peso final}

El análisis de varianza para el peso final muestra diferencias estadísticas $(p \leq 0,05)$ entre los tratamientos, el coeficiente de variación en esta etapa de la investigación fue de 
$4,91 \%$. Según los resultados de la prueba de Duncan, los tratamientos T $(30,11 \mathrm{~g})$ y E2 $(29,33 \mathrm{~g})$ son estadísticamente iguales y se diferencian de los tratamientos E1 $(34,60 \mathrm{~g})$ y E3 $(27,21 \mathrm{~g})$. El tratamiento que alcanzó mayor peso final fue el E1 $(34,59 \mathrm{~g})$ en los 112 días de investigación, este resultado sugiere que la adición de $2 \%$ del alga Ascophyllum nodosum en la alimentación de alevines de trucha es una cantidad adecuada permite ganar un mayor peso en relación con los demás tratamientos. Las comparaciones ortogonales en peso final muestran diferencias estadísticas entre E1 (34,6 g) versus E2, E3 $(28,27 \mathrm{~g})$ y es mejor el uso de $2 \%$ de adición de alga; mientras que al comparar E2 versus E3 resulta mejor E2 con 2,12 gramos sobre E3.

\subsubsection{Conversión alimenticia}

La conversión alimenticia presenta diferencias estadísticas entre los tratamientos $(p \leq 0,05)$ con un coeficiente de variación de 5,33\%. Duncan establece dos rangos, en el primero se ubican los tratamientos E1, E2, E3 que son los que contienen suplemento alga Ascophyllum nodosum con una mejor conversión alimenticia $(0,19)$ y en el segundo lugar se ubica el tratamiento testigo con una conversión de $(0,21)$; la comparación ortogonal entre T vs. E1, E2, E3 corrobora al resultado calculado por el método de Duncan. Lo descrito anteriormente permite decir que para obtener una mejor conversión alimenticia es recomendable adicionar un suplemento alimenticio en base al alga Ascophyllum nodosum.

\subsubsection{Mortalidad}

La prueba de chi cuadrado sugiere una relación estadística entre los tratamientos y el número de tilapias muertas con un p-valor $<0,0001$ y con 3 grados de libertad. Se puede notar que el mejor porcentaje de mortalidad es para E1 con 4,60\% (2\% suplemento) respecto a T con 8,53\%, E2 con 14,80\% (2,5\% suplemento y E3 con 12,07\% (3\% suplemento). Probablemente, el menor porcentaje obtenido por el T2 (2\% suplemento) se deba a que este consiguió de alguna manera mantener cierta estabilidad de la formulación del balanceado, con lo que pudo conseguir una mejor resistencia de los alevines a los factores adversos.

Tabla 4. Efecto de la influencia del alga Ascophyllum nodosum como promotor de crecimiento en la etapa de alevinaje de la trucha Arco Iris Oncorhynchus mykiss en la variable mortalidad.

\begin{tabular}{lccc}
\hline Tratamiento & Muertos & Vivos & Total \\
\hline T & $128(8,53 \%)$ & $1372(91,47 \%)$ & 1500 \\
E1 & $69(\mathbf{4}, 60 \%)$ & $1431(95,40 \%)$ & 1500 \\
E2 & $222(14,80 \%)$ & $1278(85,20 \%)$ & 1500 \\
E3 & $181(12,07 \%)$ & $1319(87,93 \%)$ & 1500 \\
Total & 600 & 5400 & 6000 \\
\hline \multicolumn{1}{c}{ Chi cuadrado de Pearson: 97,7} & GL: 3 & p-valor: $<0,0001$ \\
\hline
\end{tabular}

El resultado obtenido sobre mortalidad se corrobora con los estudios realizados por Acadian Seaplants y citado por Summer Zone, (2013) donde obtienen mejores resultados con el $2 \%$ de suplemento nutricional en peces, principalmente en lo referente a la resistencia de Aeromona salmonicida.

\subsubsection{Análisis de costos}

El costo por kilogramo de peso vivo producido en cada uno de los tratamientos fue el siguiente: $\$ 35,24$ para el E1; $\$ 42,05$ el T; $\$ 44,80$ E2 y el E3 $\$ 49,77$. El tratamiento E1 al finalizar la investigación presentó una baja mortalidad, mejor ganancia de peso diaria y 
peso final. Estos parámetros antes mencionados determinaron que el costo por kilogramo de carne producida se reduzca considerablemente en relación con los demás tratamientos.

\subsection{Evaluación del alga Ascophyllum nodosum como estimulante del sistema inmunitario de alevines de trucha Arco Iris ante desafíos patógenos}

No existió mortalidad de alevines después de la inoculación de la cepa pura del hongo Saprolegnia sp., en todos los tratamientos, esto puede atribuirse al buen manejo en cuanto a bioseguridad y nutrición durante la investigación.

\section{Conclusiones y recomendaciones}

El tratamiento que mejores resultados presentó en relación a las variables en estudio fue el E1 (98\% de alimento y $2 \%$ de alga Ascophyllum nodosum), proporcionó mejor asimilación del alimento y mayor resistencia a factores adversos que afrontan los alevines de trucha Arco Iris en sus primeros días de vida.

El alga Ascophyllum nodosum administrada en la dieta alimenticia en alevines de la trucha Arco Iris (2\%, 2,5\% y $3 \%)$, proporcionaron una excelente resistencia al desafío micótico con Saprolegnia sp., esto se comprobó con la ausencia de mortalidad de alevines durante los 15 días de observación a partir de la inoculación, demostrando la eficiencia del alga a la resistencia de enfermedades comunes.

Finalizada la investigación se recomienda utilizar el alga Ascophyllum nodosum al $2 \%$ como suplemento nutricional en la alimentación de alevines y en las diferentes etapas de desarrollo de la trucha Arco Iris, realizar un estudio más profundo del metabolismo de los alimentos, de la eficiencia del alga Ascophyllum nodosum en alevines respecto a otros desafíos de enfermedades.

\section{Bibliografía}

ACADIAN SEAPLANTS, (2017). La comida de Kelp (Kelp meal) para animales de ACADIAN SEAPLANTS. Recuperado el 01 de diciembre del 2017, de http://www.acadianseaplants.com/es/animals

CENTRO EGIPCIO INTERNACIONAL PARA LA AGRICULTURA, (1994). Curso sobre el desarrollo de la Piscicultura. Cairo, CEIA. pp. 15

Conroy, D (1991). Principales enfermedades de los salmónidos; una guía de diagnóstico y control para el biólogo. 3a . ed. Zaragoza, Acribia. pp. 120

Di Rienzo J, Balzarini M, Casanoves F, González L, Tablada M, Guzmán W. y Robledo CR. 2016. InfoStat. Software estadístico. Universidad Nacional de Córdoba. Argentina.

FAO, (2017). Papel de la FAO en la acuicultura. Recuperado el 01 de diciembre del 2017, de http://www.fao.org/aquaculture/es/

Imaki, A (1987). Introducción a la Crianza de Trucha Arco Iris. La Paz, Agencia de Cooperación Internacional del Japón (JICA) pp. 41-42-43-44

Niwa, Y. y Zeballo, A (1990). Guía de enfermedades de la trucha Arco Iris. La Paz, Agencia de Cooperación Internacional del Japón (JICA). pp. 32-33-34.

Nilubol, K. y Yuasa, K, (1998). Mycoscience: Morphological aspects of Saprolegnia sp., isolated from pejerrey. Tokyo (Japón). pp. 365-366-367.

Summer Zone, (2017). Programas de orgánicos Ecuador Summer Zone. Extraído el 1 de mayo del 2017. Disponible en http://www.organicosecuador.com/nosotros 